\title{
Electromagnetic Duality, Charges, Monopoles, Topology, ...
}

\author{
Juan A. Mignaco \\ Inst. de Física, Univ. Fed. do Rio de Janeiro \\ 21945-970, Rio de Janeiro, RJ, Brazil \\ E-mail:mignaco@if.ufrj.br
}

Received on 25 January, 2001

\begin{abstract}
In this talk we review the subject of electromagnetic duality, and related ones, as "normal" electric and magnetic charges, being different from monopoles (magnetic or electric), the topological content of monopoles, surface terms, Dirac strings and chiral structure in the inclusion of massless spinor field and its relation to torsion. We limit our discussion to the simplest, Heaviside electromagnetic duality, include some links with the nonabelian gauge theories and make no comment on the use of this concept in string theories. Old and recent contributions on the subject are presented and discussed, and possible lines of research will hopefully be indicated.
\end{abstract}

\section{Introduction}

In this talk I intend to review the notion of electromagnetic duality in its simplest forms. My views on the subject result from a long standing collaboration with Prof. Carlos A. P. Galvão first at CBPF and now at $\mathrm{UnB}$ (with several articles in preparation, and bitter discussions with referees), and numerous pleasant conversations with Prof. Clovis Wotzasek. Given the huge amount of paper in the literature on the subject, I can't think to have covered all the available material, and many will find that important references were not given enough relevance. I apologize too for those here at São Lourenço and elsewhere whose own contributions in their opinion didn't receive the appropriate consideration.

The concept of duality has received prominent attention in modern gauge theories. It provides useful tools to construct solutions to the field equations, namely, those which are self-dual or anti-self-dual, or allows to study regimes of the theory which prevent the use of perturbation expansions. These features seem particularly important for recent developments in string theory and its derivatives,such as branes, M-theory [1]. I shall not deal with these advanced matters.

Duality in classical electromagnetic theory was discovered by Heaviside [2] a century ago for the Maxwell equations in vacuum. He saw that

$$
\left.\begin{array}{l}
\nabla \wedge \mathbf{E}=-\frac{\partial \mathbf{B}}{\partial t} \\
\nabla \wedge \mathbf{B}=\frac{1}{c^{2}} \frac{\partial \mathbf{E}}{\partial t}
\end{array}\right\} .
$$

exchange among themselves under the replacements

$$
\mathbf{E} \rightarrow-c \mathbf{B}, \quad c \mathbf{B} \rightarrow \mathbf{E} .
$$

This symmetry of the system, duality (we shall refer to it as Heaviside duality), originated a lot of speculation about its meaning: is the electric field to be considered equivalent to the magnetic induction field, and the reverse?.

It seems that Larmor [3] generalized Heaviside duality to a continuous transformation:

$$
\left.\begin{array}{rl}
\mathbf{E}_{\eta} & =\mathbf{E} \cos \eta-c \mathbf{B} \sin \eta \\
c \mathbf{B}_{\eta} & =\mathbf{E} \sin \eta+c \mathbf{B} \cos \eta
\end{array}\right\}, 0 \leq \eta \leq \frac{\pi}{2} .
$$

This emphasizes that there is complete ambiguity or equivalently, continuous freedom, in the choice of electric and induction fields for the radiation fields.

With the advent of nonabelian gauge theories for elementary particle physics, a lot of work in Physics and Mathematics has been performed to clarify the meaning and applicability of duality, as exposed above, or in its modern nonabelian versions.

Let us recall that the original fields in the Maxwell equations have electric charges or currents and/or time varying fields as their sources. Even in vacuum, electric fields are considered the ones accelerating electric charges parallel to their direction, whereas magnetic fields provide transverse acceleration for electric charges. Magnetic materials are related to elementary magnetic dipoles but single isolated magnetic charges have never been observed.

Dirac [4], motivated by the need to explain the quantization of the electric charges, introduced magnetic monopoles in this framework to provide sources 
for the magnetic induction field, i. e.,

$$
\nabla \cdot \mathbf{B}=\mu_{0} g \delta(\mathbf{x})
$$

To preserve the relation with the magnetic vector potential,

$$
\mathbf{B}=\nabla \wedge \mathbf{A}
$$

a filamentary solenoid had to extend from the position of the magnetic charge to infinity. In the quantum regime, the unobservability of this determined the famous Dirac relation between the electric charge of a test particle and the strength of the monopole field ${ }^{1}$ :

$$
q g=2 \pi n \frac{\hbar}{\mu_{0}} .
$$

The topological characterization of the problem [6][7] was established almost half a century later. It is usually strongly assumed that the introduction of magnetic monopoles is related to Heaviside duality.

There remain unwanted aspects of the theory at the electromagnetic level. Standing high is the fact that the lagrangian of the theory changes sign under duality, that is, Heaviside duality is a symmetry for the equations of motion but not for the lagrangian providing them. Neglecting sources,

$$
L\{\mathbf{E}, \mathbf{B}\}=\frac{\epsilon_{0}}{2} \int d^{3} x\left(\mathbf{E}^{2}-c^{2} \mathbf{B}^{2}\right)
$$

Let me list the works which somehow were landmarks on this subject.

- Dirac's 1947 article proposing a string theory for monopoles [8]

- The two potential formalism by Nisbet in 1955 [9] and Cabibbo and Ferrari in 1962 [10]

- Röhrlich's article on the impossibility of a classical local theory with monopoles [11]

- Schwinger contributions in 1966-75 [12]

- Zwanziger formulations in field theory 1968-72 [13]

- The formalism of fibre bundles for the magnetic monopole set forth by Greub and Petry [6] and Wu and Yang [7]

- Deser and Teitelboim construction of a non local duality generator in 1976 [14]

- Olive and Montonen work in 1977 about magnetic monopoles in gauge theories [15]

- Schwarz and Sen work in 1994 with a non-Lorentz invariant lagrangian [16]
- Seiberg and Witten use of duality in supersymmetric string theories in 1995 [17]

In the following, I shall dwell on some of this work, a criticism will be addressed when considered necessary and shall try to frame them in a common realm.

\section{Some preliminaries}

The talk is about a specific symmetry. What we understand under this name is a precise dynamical idea: a system exhibits a symmetry when under its action the properties of the system remain unaltered. Conversely, a symmetry is an action which cannot discriminate differences on a given physical system.

To see that it is a dynamical concept, take for instance the hydrogen atom: as long as your spectroscope is not able to separate the fine structure, it looks symmetric under the usual space rotation symmetry. Once the spin of the particles is taken into account, this symmetry doesn't hold anymore.

The breaking of a symmetry results in an ordering of the resulting physical characteristics: it may grossly preserve it, as in the preceding case of the hydrogen atom, or disturb it deeply, as the electroweak symmetry breaking.

To fix notations, as shown before vectors in threespace will be denoted by boldface characters, $\mathbf{A}, \mathbf{j}, \mathbf{E}$, $\mathbf{B}, \ldots$. ; in what follows, four dimensional tensorial quantities will be denoted by italics, $A, j, F, \ldots$; and finally, differential forms by calligraphic letters, $\mathcal{A}, \mathcal{J}$ $, \mathcal{F}, \ldots$.

The field intensities $\mathbf{E}$ and $c \mathbf{B}$ will be associated to the field strength tensor, $F^{\mu \nu}$, and a two-form differential form in four-dimensional spacetime:

$$
\begin{aligned}
\mathcal{F} & =\frac{1}{2} F_{\mu \nu} d x^{\mu} \wedge d x^{\nu} \\
& =F_{0 k} d x^{0} \wedge d x^{k}+F_{i j} d x^{i} \wedge d x^{j}
\end{aligned}
$$

The usual inhomogeneous Maxwell equations read (repeated indices indicate sums)

$$
\partial_{\mu} F^{\mu \nu}=\mu_{0} c j^{\nu}
$$

with $j^{\mu} \equiv(c \rho, \mathbf{j})$. From the homogeneous equations, one has Bianchi identity for $F$ :

$$
\partial_{\lambda} F_{\mu \nu}+\partial_{\mu} F_{\nu \lambda}+\partial_{\nu} F_{\lambda \mu}=0
$$

Using differential forms, we have for Maxwell equations

$$
\left.\begin{array}{rl}
\delta \mathcal{F} & =-\mu_{0} c \mathcal{J}_{e} \\
d \mathcal{F} & =0
\end{array}\right\}
$$

\footnotetext{
${ }^{1}$ It is worth pointing that in the expression appears a quantum of flux which is half the one needed for the unobservability of the solenoid in the Ehrenberg-Siday-Aharonov-Bohm "experimental" setup.

Curiously putting for $q$ the value of the electronic charge, and $n$ taken as two, the resulting value for $g$ is the inverse of the constant for the Josephson effect, $K_{J}=2 e / h=483598 G H z / V=1 / g(n=2)[5]$
} 
The symbols $d$ and $\delta$ correspond to differential operators, the generalizations for antisymmetric tensors of the usual curl and divergence operators in three space. Given a differential p-form $\omega$

$$
\omega=w_{i_{1} \ldots i_{p}} d x^{i_{1}} \wedge \cdots \wedge d x^{i_{p}}
$$

the action of $d$ is given by

$$
d \omega=\partial_{i_{k}} w_{i_{1} \cdots i_{p}} d x^{i_{k}} \wedge d x^{i_{1}} \wedge \cdots \wedge d x^{i_{p}}
$$

that is, it produces a differential $(p+1)$-form from a differential $p$-form . The other operator performs the opposite way, relating a differential $p$-form with a differential $(p-1)$-form, as

$$
\delta \omega=-g^{i_{k} i_{l}} \partial_{i_{k}} w_{i_{1} \cdots i_{p}} d x^{i_{1}} \wedge \cdots\left(\operatorname{nod}^{i_{l}}\right) \cdots \wedge d x^{i_{p}} .
$$

Another operation which will play a rôle in what follows is the Hodge duality operator for differential forms, $*$, which relates differential $p$-forms with differential $(n-p)$-forms, $n$ being the total dimension of the manifold, in most of our talk the four-dimensional spacetime. Acting on the base monomials it gives:

$$
\begin{aligned}
& * d x^{\mu_{1}} \wedge \cdots \wedge d x^{\mu_{p}}= \\
& \frac{1}{(n-p) !} g^{\mu_{1} \nu_{1}} \cdots g^{\mu_{p} \nu_{p}} \varepsilon_{\nu_{1} \cdots \nu_{p} \nu_{p+1} \cdots \nu_{n}} d x^{\nu_{p+1}} \wedge \cdots d x^{\nu_{n}}
\end{aligned}
$$

$p$-forms into a Hilbert space, defining properly an internal product. With this definition, the differential operators $d$ and $\delta$ appear as adjoint to the other.

It is easy to show that for differential 2-forms, Hodge duality applied to $\mathcal{F}$ produces the result of Heaviside duality for the fields $(\mathbf{E}, c \mathbf{B})$.

In the real world where electrons strike on $\mathrm{TV}$ screens and electric generators light the nights of modern times, the presence of sources, electric charges and current densities, abrogates the validity of Heaviside duality. Let us recall the complete set of Maxwell equations:

$$
\left.\begin{array}{rl}
\nabla \cdot \mathbf{D} & =\rho_{e} \\
\nabla \cdot \mathbf{B} & =0 \\
\nabla \wedge \mathbf{E} & =-\frac{\partial \mathbf{B}}{\partial t} \\
\nabla \wedge \mathbf{H} & =\mathbf{j}_{e}+\frac{\partial \mathbf{D}}{\partial t}
\end{array}\right\}
$$

Electric charges and the invariance of the equations under Lorentz transformations show that the electric and magnetic fields cannot be taken on the same footing. In fact, magnetic fields are a kind of "kinematic" effect from charges in motion, in general. Only for radiation fields both electric and magnetic induction have equivalent properties.

In terms of four-dimensional field tensors, in vacuum, we have eqs. (10) and (11), and we may write them in terms of differential forms as eqs. (12). For what follows, let me write the latter in its dual version:

$$
\left.\begin{array}{rl}
\delta * \mathcal{F} & =0 \\
d * \mathcal{F} & =-\mu_{0} c * \mathcal{J}_{e}
\end{array}\right\}
$$

In the following, having in mind the extensions of the former equations into a generalized duality framework, I shall try to analyze the possible answers to some appealing questions:

- Does the generalization of Heaviside duality imply exclusively the introduction of Dirac's magnetic monopoles?

- Should the magnetic field of a magnetic charge be the same as obtained from electric currents?

- Should the generalized Heaviside duality to be taken as identical to Hodge duality for differential forms?

- Is the present physical world emerging from a breaking of Heaviside duality ?

\section{Duality without sources}

The transformation devised by Heaviside has a discomforting consequence already at the free-field level. The energy density of the electromagnetic field remains invariant:

$$
u_{e m}=\frac{1}{2} \epsilon_{0}\left(\mathbf{E}^{2}+c^{2} \mathbf{B}^{2}\right)
$$

whereas the action density

$$
s=\frac{1}{2} \epsilon_{0}\left(\mathbf{E}^{2}-c^{2} \mathbf{B}^{2}\right)
$$

changes sign. Heaviside's transformation is a symmetry for the equations of motion, but not for the lagrangian generating them.

There are two most accepted ways of overcoming this, the first one proposed in 1976 by Deser and Teitelboim [14], and almost two decades later the second by Schwarz and Sen [16].

Deser and Teitelboim looked for a generator for the Heaviside duality transformations that leaves the energy invariant. This they succeeded to find, and proposed an expression local in time:

$$
G=\frac{1}{2} \int d^{3} x\left(\mathbf{E} \cdot \nabla^{-2} \nabla \wedge \mathbf{E}+c^{2} \mathbf{B} \cdot \nabla^{-2} \nabla \wedge \mathbf{B}\right)
$$

The fact that one has to reccur to a generator nonlocal in space is somewhat ugly.

Schwarz and Sen [16] went along different lines of thought. They proposed an action with two sets of fields and potentials $(\alpha=1,2)$ :

$$
S=-\frac{1}{2} \int d^{4} x\left(B^{(\alpha) i} \mathcal{L}_{\alpha \beta} E^{(\beta)}{ }_{i}+B^{(\alpha) i} B^{(\alpha)}{ }_{i}\right)
$$


with the matrix $\mathcal{L}$ being

$$
\|\mathcal{L}\|=\left(\begin{array}{rr}
0 & 1 \\
-1 & 0
\end{array}\right) .
$$

The fields are defined through

$$
\begin{aligned}
E_{i}^{(\alpha)} & =\partial_{0} A^{(\alpha)}{ }_{i}-\partial_{i} A^{(\alpha)}{ }_{0}, \\
B^{(\alpha) i} & =\varepsilon^{i j k} \partial_{j} A^{(\alpha)}{ }_{k}, \alpha=1,2 ; 1 \leq i j k \leq 3 .
\end{aligned}
$$

Taking into account the gauge freedom of the theory they set

$$
A_{0}^{(\alpha)}=0 \text {. }
$$

One of the equations of motion is

$$
B_{k}^{(2)}=E_{k}^{(1)}
$$

and it allows to recover the usual Maxwell equations for the $\alpha=1$ fields. The duality for the potentials has a local expression

$$
A^{(\alpha)}{ }_{\mu}=\mathcal{L}_{\alpha \beta} A^{(\beta)}{ }_{\mu}
$$

the only flaw being the apparent Lorentz noninvariance of the equations. They were able to prove that Lorentz invariance holds.

Pakman [20] observed that for the free fields the equations of motion read:

$$
\partial_{\mu} F^{\mu \nu}\left[A_{\mu}\right]=0,
$$

with

$$
F_{\mu \nu}=\partial_{\mu} A_{\nu}-\partial_{\nu} A_{\mu} .
$$

The last equation implies the Bianchi identities, which, written in terms of the Hodge dual of $F_{\mu \nu}$ read

$$
\partial^{\mu} * F_{\mu \nu}\left[A_{\mu}\right]=0 \text {. }
$$

He proposes to introduce a new potential for the dual tensor, $Z_{\mu}$,

$$
* F_{\mu \nu}\left[Z_{\mu}\right]=\partial_{\mu} Z_{\nu}-\partial_{\nu} Z_{\mu}
$$

and writing the action in terms of the dual tensor, the equation of motion is now

$$
\partial^{\mu} * F_{\mu \nu}=0
$$

whereas the corresponding Bianchi identities (the former equations of motion) are

$$
\partial_{\mu} F^{\mu \nu}\left[Z_{\mu}\right]=0 \text {. }
$$

We shall show below other examples on this line of thought. The Schwarz-Sen action may now be written in terms of the two four potentials, $A_{\mu}, Z_{\mu}$, and it is a kind of interpolation for the situations described above. Besides, Pakman proposes a continuous $U(1)$ generalization using complex fields and potentials.
Girotti [21] analyzed the quantization of this model in the Coulomb gauge using the Dirac bracket formalism. He showed that the quantized theory propagates massless particles, and explicitly demonstrated that the theory is genuinely Lorentz invariant. In fact, I see two massless particles propagating.

Dwelling deeper into the subject, Girotti, Marcelo Gomes, Victor Rivelles and Adilson da Silva [22] showed that the formalism of Schwarz and Sen at the quantum level is equivalent to the proposal of Deser and Teitelboim, and of course, both reproduce the quantum theory of the Maxwell field. They obtained the charge that generates the infinitesimal duality transformation:

$$
\begin{gathered}
Q=-\frac{1}{2} \int d^{3} x \epsilon^{i j k}\left(\partial_{j} A^{(\alpha)}{ }_{i}\right) A^{(\alpha)}{ }_{k} \\
=\frac{1}{2} \int d^{3} x B^{(\alpha) k} A^{(\alpha)}{ }_{k} .
\end{gathered}
$$

Its relation with a Chern-Simons term guarantee that it is gauge invariant.

An interesting interpretation of the work by Schwarz and Sen was shown to me by Clóvis Wotzasek. Take the oscillator decomposition of modes at a given frequency, $\omega=\omega(k)$. The lagrangian density may be written as

$$
L=p \dot{x}-\frac{1}{2} p^{2}-\frac{1}{2} x^{2}
$$

and write $p \dot{x}=\frac{1}{2}(p \dot{x}-x \dot{p})$, then redefine $p=x_{1}$ and $x=x_{2}$ and one recovers the Schwarz and Sen form of the lagrangian. The procedure is a reparametrization, a relabelling of the potential fields.

Other proposals to overcome the problem of covariance are the introduction of an infinite number of auxiliary fields [18] or a non-polynomial lagrangian of auxiliary fields [19].

In previous editions of this meeting we have heard contributions to this problem from Girotti and our colleagues of USP, and others by Clóvis Wotzasek, Rabin Banerjee and collaborators. The latter study the generalization of Heaviside duality to spacetimes of higher (even) dimensions. The relevant extended electromagnetic theory is the one defined through antisymmetric tensors having $2(n-1)$ components ( $n$ being as before the number of spacetime dimensions). For $n=4 k, k$ :integer, the group implementing duality is $Z_{2}$, whereas for dimension $n=4 k+2$, the group is $S O(2)$. By introducing "external" additional variables, they find a "duality of duality", in the sense that when the group is $Z_{2}$ ( $n=4 k$ for the normal variables), the "external" ones transform as $S O(2)$, and the opposite in the other dimensions [23]. The new "duality" exchanges internal and external variables ${ }^{2}$.

Notice that all these works do not take for granted that the electromagnetic fields introduced through new potentials are indeed the same as the old ones. They either propose to incorporate the symmetry in electromagnetism, suitably generalized, or a new theory where the symmetry is implemented.

\footnotetext{
${ }^{2}$ In conversations with Clóvis we have speculated that their results may be somehow a generalization of Schwarz-Sen
} 


\section{Including sources}

The generalization of Maxwell equations with magnetic charges and currents is $[12,24,26]$ :

$$
\left.\begin{array}{rl}
\nabla \cdot \mathbf{D} & =\rho_{e} \\
\nabla \cdot \mathbf{B} & =\mu_{0} \rho_{m} \\
\nabla \wedge \mathbf{E} & =-\frac{1}{\epsilon_{0}} \mathbf{j}_{m}-\frac{\partial \mathbf{B}}{\partial t} \\
\nabla \wedge \mathbf{H} & =\mathbf{j}_{e}+\frac{\partial \mathbf{D}}{\partial t}
\end{array}\right\}
$$

There are no homogeneous equations and we have two conserved currents:

$$
\left.\begin{array}{rl}
\nabla \cdot \mathbf{j}_{e}+\frac{\partial \rho_{e}}{\partial t} & =0 \\
\nabla \cdot \mathbf{j}_{m}+\frac{1}{c^{2}} \frac{\partial \rho_{m}}{\partial t} & =0
\end{array}\right\}
$$

Notice that the magnetic charges are related to the flux of the magnetic induction field. Calling $Q_{m, V}$ the total magnetic charge in a volume $V$, and $S$ the surface at the boundary of $V$, then

$$
\oint_{S} \mathbf{B} \cdot d \mathbf{S}=\mu_{0} Q_{m, V}
$$

The equations implicitly identify as the same quantity the fields generated by electric charges and magnetic currents, for the electric field, and generated by magnetic charges and electric currents for the magnetic induction field. These equations are invariant under a generalization of the Heaviside transformations:

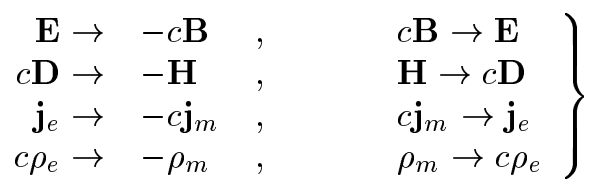

The free field equations continue to have two degrees of freedom. The addition of sources do not change the empty space counting of degrees of freedom, and the added invariance reduces the number with sources.

In this case, it seems pointless to speak about Bianchi identities, which result in the introduction of potentials.

In terms of differential forms, we have now

$$
\left.\begin{array}{rl}
\delta \mathcal{F} & =-\mu_{0} c \mathcal{J}_{e} \\
d \mathcal{F} & =-\mu_{0} c \mathcal{J}_{m}
\end{array}\right\}
$$

where $\mathcal{J}_{e}$ is the one-form that corresponds to the electric current density and $\mathcal{J}_{m}$ is a three-form having as components the magnetic current density vector. The different meanings of the currents is in evidence. If, as a useful exercise, one introduces the Heaviside transformations $(\star)$ in both members of these equations, one finds

$$
\left.\begin{array}{rl}
* \mathcal{F} & =\star \mathcal{F} \\
* \mathcal{J}_{e} & =\star \mathcal{J}_{m} \\
* \mathcal{J}_{m} & =-\star \mathcal{J}_{e}
\end{array}\right\}
$$

The important point is that Hodge duality is a geometrical transformation, the physical content of the equations is the result of imposing Heaviside duality for the Maxwell equations, no matter in which form one expresses them. When the equations satisfy Heaviside duality, Maxwell equations receive a Hodge dual symmetric form.

To introduce potentials, it is enough to switch off the electric charge and current densities. The first and fourth equations become homogeneous, and suggest the introduction of a magnetic (pseudo)scalar potential, $\psi$, and an electric (pseudo)vector potential, $\mathbf{W}$, with the natural definition for the fields:

$$
\left.\begin{array}{l}
\mathbf{E}=-\nabla \varphi-\frac{\partial \mathbf{A}}{\partial t}+\nabla \wedge \mathbf{W} \\
\mathbf{B}=-\nabla \psi+\frac{1}{c^{2}} \frac{\partial \mathbf{W}}{\partial t}+\nabla \wedge \mathbf{A}
\end{array}\right\} .
$$

There is no conflict with usual three-dimensional vector analysis in taking the divergence of these fields, one arrives at ordinary Poisson equations for the potentials $\varphi$ and $\psi$ in each case. The imposition of Heaviside duality is achieved with no magnetic monopoles.

Since Maxwell equations are invariant under Heaviside transformations, the potentials have correspondingly to satisfy the following relations:

$$
\left.\begin{array}{cl}
\mathbf{W} \rightarrow-c \mathbf{A} & , \quad c \mathbf{A} \rightarrow \mathbf{W} \\
\varphi \rightarrow-c \psi & , \quad c \psi \rightarrow \varphi
\end{array}\right\}
$$

Since applying twice Heaviside transformations one gets a global minus sign for all quantities involved, we see that Heaviside duality IS NOT identical to Hodge duality for differential forms, it coincides just for the fields, not for the currents and potentials.

The relativistic formulation however proves to be troublesome. Define the following four-vectors:

$$
\left.\begin{array}{rl}
A_{\mu} & \equiv\{\varphi,-c \mathbf{A}\} \\
j_{e \mu} & \equiv\left\{c \rho_{e},-\mathbf{j}_{e}\right\} \\
W_{\mu} & \equiv\{c \psi, \mathbf{W}\} \\
j_{m \mu} & \equiv\left\{\rho_{m}, c \mathbf{j}_{m}\right\}
\end{array}\right\}
$$

Calling

$$
A_{\mu \nu}=\partial_{\mu} A_{\nu}-\partial_{\nu} A_{\mu} \quad, \quad W_{\mu \nu}=\partial_{\mu} W_{\nu}-\partial_{\nu} W_{\mu}
$$

one writes the tensor for the field intensities as

$$
F_{\mu \nu}=A_{\mu \nu}+\varepsilon_{\mu \nu \rho \sigma} W^{\rho \sigma}
$$

The relativistic invariant lagrangian density having the extended Maxwell equations with Heaviside duality as a symmetry is

$$
\mathcal{L}_{A+W}=-\frac{1}{4} \epsilon_{0} F_{\mu \nu} F^{\mu \nu}-\frac{1}{c} j_{e \mu} A^{\mu}-\frac{1}{c} j_{m \mu} W^{\mu} .
$$


The first term in the above expression merits a closer look. It is

$$
F_{\mu \nu} F^{\mu \nu}=A_{\mu \nu} A^{\mu \nu}-W_{\mu \nu} W^{\mu \nu}+\frac{1}{3} \varepsilon_{\mu \nu \rho \sigma} A^{\mu \nu} W^{\rho \sigma} .
$$

The last term is a "surface term", and it it should be neglected under the usual assumptions. Its consequences will be discussed in what follows.

The canonical momenta are:

$$
\begin{aligned}
\Pi_{k}^{(A)} & =\epsilon_{0} E_{k} \\
\Pi_{k}^{(W)} & =-\epsilon_{0} c B_{k}
\end{aligned}
$$

Under Heaviside duality, they transform properly:

$$
\Pi_{k}^{(W)} \rightarrow-\Pi^{(A)}{ }_{k}, \quad \Pi_{k}^{(A)} \rightarrow \Pi_{k}^{(W)} .
$$

As before, the lagrangian changes sign under Heaviside duality. The corresponding full canonical hamiltonian reads:

$$
\begin{aligned}
\mathcal{H}_{A+W} & =\frac{1}{2} \epsilon_{0}\left(\frac{1}{\epsilon_{0}} \Pi_{k}^{(A)}+\varepsilon_{0 k i j} W_{i j}\right)^{2} \\
& -\frac{1}{2} \epsilon_{0}\left(\frac{1}{\epsilon_{0}} \Pi^{(W)}{ }_{k}+\varepsilon_{0 k i j} A_{i j}\right)^{2} \\
& +A_{0}\left(\partial_{k} \Pi^{(A)}{ }_{k}\right)+W_{0}\left(\partial_{k} \Pi^{(W)}{ }_{k}\right) \\
& +\frac{1}{4} \epsilon_{0} A_{i j}{ }^{2}-\frac{1}{4} \epsilon_{0} W_{i j}{ }^{2}+\frac{1}{c} j_{e \mu} A^{\mu}-\frac{1}{c} j_{m \mu} W^{\mu}
\end{aligned}
$$

Under Heaviside duality, the hamiltonian too changes sign. The only way out for this situation in the quantized version of the theory may be the reversal of either space or time. The integrated hamiltonian being the fourth total momentum component, it seems that the last, time reversal, is the appropriate one.

Notice the important point that in this formalism it is implicit that the electric field and magnetic induction field generated by electric or magnetic charges and current densities are the same. When this translates at the potential dependence, we face this troublesome situation.

For what regards the gauge invariance properties of the theory, it is invariant under $[9,27]$

$$
\left.\begin{array}{rl}
A_{\mu} \rightarrow A^{\prime}{ }_{\mu} & =A_{\mu}+\partial_{\mu} \Lambda+\partial^{\lambda} P_{\lambda \mu} \\
W_{\mu} \rightarrow W^{\prime}{ }_{\mu} & =W_{\mu}+\partial_{\mu} \Xi+\varepsilon_{\mu \nu \rho \sigma} P^{\rho \sigma}
\end{array}\right\}
$$

with $\square P_{\mu \nu}=0$. The theory displays a kind of $U(1) \otimes U(1)$ gauge symmetry provided the "surface" term in the lagrangian is neglected and $P_{\mu \nu}$ is taken zero.

The continuous duality transformation holds for the fields and related quantities. Indeed, as pointed by Jackson [28] this shows that the direction which is taken as magnetic or electric in the field space is arbitrary. The lagrangian, however, transforms as:

$$
\mathcal{L}_{A+W, \eta}=\mathcal{L}_{A+W} \cos 2 \eta+\mathcal{L}^{\prime} \sin 2 \eta
$$

where $\mathcal{L}^{\prime}$ is an interesting intermediate expression:

$$
\mathcal{L}^{\prime}=\frac{1}{4} \epsilon_{0} A_{\mu \nu} W^{\mu \nu}-\frac{1}{8} \epsilon_{0} \varepsilon^{\mu \nu \rho \sigma}\left(A_{\mu \nu} A_{\rho \sigma}+W_{\mu \nu} W_{\rho \sigma}\right)
$$

which is a pseudo-scalar quantity under parity transformations. The last terms reminds of the expressions obtained from the calculation of the $U(1)$ electromagnetic anomaly.

\section{The monopoles}

The question of magnetic monopoles appears often linked with electromagnetic duality. In several contributions, Olive and collaborators [15, 29] proposes a general theory including particles (carriers of the electric charges) and monopoles. We have heard about this work here and at the "Jorge André Swieca" school exposed by Marco Aurelio Kneipp [30].

The simplest version of monopole is a magnetic point charge at the origin (4) :

$$
\nabla \cdot \mathbf{B}=\mu_{0} g \delta(\mathbf{x}) .
$$

By Gauss's theorem, the flux of $\mathbf{B}$ around that charge is:

$$
\Phi=\oint \mathbf{B} \cdot d \mathbf{S}=\mu_{0} g
$$

Taking, by assumption,

$$
\mathbf{B}=\nabla \wedge \mathbf{A}
$$

one has

$$
\nabla \cdot \mathbf{B}=\nabla \cdot(\nabla \wedge \mathbf{A})=0 .
$$

In his 1947 article, Dirac [8] recognizes the incompatibility of both requirements. To write a magnetic potential for the monopole, a Dirac string is needed. To guarantee unobservability of the string by charged particles, the electric charge and the monopole charge should satisfy Dirac's condition. What Dirac proposes is an enlargement that produce a dynamics to the string. He writes

$$
F_{\mu \nu}=A_{\mu \nu}+\sum\left(G^{\dagger}\right)_{\mu \nu}
$$

The sum is over all the world sheets swept by a Dirac string. The result for $G_{\mu \nu}$ he obtains is

$$
G_{\mu \nu}(x)=g \iint d \tau d \sigma\left[\frac{\partial y_{\mu}}{\partial \tau} \frac{\partial y_{\nu}}{\partial \sigma}-\frac{\partial y_{\mu}}{\partial \sigma} \frac{\partial y_{\nu}}{\partial \tau}\right] \delta^{4}(x-y)
$$

I understand that duality between electric charges and magnetic monopoles means, for Dirac, the exchange of the first term in the field-strength tensor with the second.

The topological approach to the theory of the magnetic monopole was due almost simultaneously to 
Greub and Petry [6] and Wu and Yang [7]. It is framed in fiber bundle language, and starts from a punctured $R^{3}$ space, which gives essentially a sphere. The magnetic potential is not globally defined, and in the transition region $(z \sim 0)$ both local definitions are related through a gauge transformation. This seems an innocent trick, but the fact is that the gauge function is a (in Dirac words) non-integrable function, i.e., an angle (an example of a closed but non- exact one-form in $R^{2}$ ).

$$
\begin{aligned}
A_{\mu} & =A_{\mu}{ }^{I}, z>0 \\
A_{\mu} & =A_{\mu}{ }^{I I}, z<0 \\
A_{\phi}{ }^{I I} & =A_{\phi}{ }^{I} \pm n \phi, z \sim 0 .
\end{aligned}
$$

The integer $n$ assumes any value. One may recall that was through a singular gauge transformation with an angle that Bocchieri and Loinger [31] eliminated the effect of the solenoid in the Ehrenberg-Siday-AharonovBohm setup.

Some time later, Ryder [37] studied the monopole as a realization of the principal bundle Hopf mapping $S^{3} \rightarrow S^{2}$. This yields a unique value, $n=2$, in agreement with previous reult by Schwinger [38]. In fact, it is not only the principal bundle which is involved for other values of $n$, but rather the related "lens spaces". These are obtained from the $S^{3}$ sphere parametrized by two complex variables

$$
\left|z_{1}\right|^{2}+\left|z_{2}\right|^{2}=1
$$

through the equivalence classes $\left(z_{1} e^{i 2 \pi k l_{1} / n}, z_{2} e^{i 2 \pi k l_{2} / n}\right)$ with $\left(k, l_{1}, l_{2}\right)$ relative prime numbers and $n$ an integer.

In the two-potential formalism presented in the previous section, if one keeps the "surface term" it contributes to the minimization

$$
\frac{\delta \mathcal{L}_{A+W}}{\delta\left(\partial^{\lambda} W^{\mu}\right)}=-\epsilon_{0} \varepsilon_{\lambda \mu \rho \sigma} A^{\rho \sigma},
$$

and yields the Bianchi identity for the potential field strength.

It is immediate that a monopole breaks Bianchi identity likewise a "normal" magnetic charge does, but makes it through a more elaborated contribution. In a contribution by P. C. R. Cardoso de Mello, S. Carneiro e M. C. Nemes, they proposed a non-local lagrangian [32] for a dual theory with two potentials. One may take a similar expression to theirs for the potentials:

$$
\begin{aligned}
A_{\mu} & =A_{(0) \mu}+\frac{1}{2} \varepsilon_{\mu \alpha \beta \lambda} \int_{P}{ }^{x} \partial^{\alpha} A_{(m)}{ }^{\beta} d y^{\lambda} \\
W_{\mu} & =W_{(0) \mu}+\frac{1}{2} \varepsilon_{\mu \alpha \beta \lambda} \int_{P}{ }^{x} \partial^{\alpha} W_{(m)}{ }^{\beta} d y^{\lambda}
\end{aligned}
$$

where the subscripts indicate no topologial structure and magnetic or electric monopole, respectively, and the latter contribute to the Euler equation.

The same mechanism produces electric monopoles, different from the usual electric charges. The minimization equation with respect to the magnetic potential has a contribution from the surface term in the lagrangian. An electric monopole would break the identity $\nabla \cdot(\nabla \wedge \mathbf{W})=0$.

For the magnetic monopole, Greub and Petry showed [6] that the compatibility of the Schrödinger equation for charged particles and the non-null value for the magnetic flux (taking the wave function as a section of a line bundle on $S^{2}$ ) limits the values for the charges through the Dirac condition and shows the existence of an integral, real, de Rham cohomology class for the manifold $M$, in our case, $S^{3} \sim S^{2} \otimes S^{1}$ (locally). In terms of differential forms, one has simultaneously $d \mathcal{F}=0(\mathcal{F}$ is a closed differential form $)$ and $\oint \mathcal{F} \neq 0(\mathcal{F}$ is not an exact form). Closed forms which are not exact expands the Hodge cohomology group. The analogous case for electric monopoles, i. e., $d * \mathcal{F}=0, \oint * \mathcal{F} \neq 0$ has been considered by Cabibbo and Ferrari [10] and Dijkgraaf [33].

The approach to duality by Olive in more than two decades is an extension of the meaning of electromagnetic duality [15]. It is inspired by previous work by Coleman [34] and Mandelstam [35] on the relation between the sine-Gordon model and the Thirring model in $1+1$ spacetime. In brief, the fermions in the Thirring model may be written as solitons of the sine-Gordon model, via a so called "duality" relation between the two models, which, besides, links the weak coupling regime in one model with the strong coupling regime in the other. This analogy has been pursued further in non-abelian theories by Olive as well as other authors $[46, ?]$.

The quanta of the field in one case are the electrically charged particles, and the solitons are magnetic monopoles. Electromagnetic duality, in this extended sense, is seen as the remnant of a non-abelian gauge symmetry, broken by the Higgs-Kibble-Brout-EnglertAnderson mechanism [36], with the HKBEA particles in the adjoint representation of the original gauge group.

A mass formula is proposed for all the quanta of the theory, which, on account of the dual symmetry proposed should not distinguish electric and magnetic charges. It is

$$
M=a \sqrt{q^{2}+g^{2}}
$$

The natural framework for the realization of these ideas in a quantum theory seems to be $N=4$ supersymmetry. Further developments led to the concepts of $\mathrm{S}$ and $\mathrm{T}$ duality widely circulating nowadays. In my opinion, the whole scheme seems plausible, but remains til now highly speculative. For a recent update on these matters, I refer to the review by Marco Aurelio [30].

\section{Massless Dirac fields sources}

Since decades the construction of a Heaviside 
dual symmetric field theory with electric and magnetic charged spinor fields has been studied [12, 13]. Fermions were in general massive, and the two-potential theory is used for the electromagnetic field. Magnetic charges are taken generally as monopoles, disregarding "normal" charges (an exception being Nisbet [9]). Curiously, properties under space inversion of the fields were mostly not accounted.

We present here a sketch of results involving massless spinor fields with "normal" electric and magnetic charges. The free spinor field lagrangian is

$$
\mathcal{L}_{\Psi}=i \hbar c \bar{\Psi} \not \supset \Psi
$$

It can be separated in chiral contributions:

$$
\mathcal{L}_{\Psi}=\mathcal{L}_{\Psi, L}+\mathcal{L}_{\Psi, R}
$$

The interaction is now introduced with both potentials of a pretense Heaviside dual electromagnetic theory via the extended covariant derivative:

$$
\mathcal{D}_{\mu}=\partial_{\mu}+i \frac{q}{\hbar c} A_{\mu}+i \frac{g}{\hbar c^{2}} \gamma^{5} W_{\mu} .
$$

The left-right chiral separation remains, with

$$
\mathcal{D}^{L, R}{ }_{\mu}=\partial_{\mu}+i \frac{q}{\hbar c} A_{\mu} \pm i \frac{g}{\hbar c^{2}} W_{\mu} .
$$

A different combination works for each chirality, and they may be qualified as "chiral" left and right potentials. They exchange under Heaviside duality transformations.

The imposition of duality restricts the values of the electric and magnetic charges to the relation:

$$
g=q c
$$

under the hypothesis that both charges are able to produce the same fields.

The left and right potentials are

$$
\begin{aligned}
& Z_{L}{ }^{\mu}=A^{\mu}+c W^{\mu} \\
& Z_{R}{ }^{\mu}=A^{\mu}-c W^{\mu}
\end{aligned}
$$

and if one rewrites the potentials $A_{\mu}, W_{\mu}$ in terms of these right and left potentials, the two-potential lagrangian (48) separates nicely in left and right parts. It appears as a $U(1)_{L} \otimes U(1)_{R}$ symmetric model (but what about the monopoles?), a sugestion put forward by Singleton [24].

The relation between spinor fields on a manifold and monopole fields have been studied on the twodimensional sphere $S^{2}$ by Jayewardena [39] for the Schwinger model, and by our group in Rio using Connes-Lott noncommutative version for the abelian theory [40]. It turns out that the number of zero modes of the Dirac operator is related to the integer defined by the Chern character. Besides, the non-trivial topology induced by the monopole field leads for the Schwinger model to the axial anomaly and a non-zero value for the condensate described by the vacuum expectation value of the fermion field, $\langle\bar{\Psi} \Psi>$.

With C. A. P. Galvão we have recalculated the axial anomaly in four (Euclidean) dimensions for the twopotential case, a calculation performed previously by Balachandran and collaborators [41]. The result is

$$
\begin{aligned}
& \frac{1}{16 \pi^{2}} \operatorname{tr}\left\{\gamma^{5} a_{2}\right\}= \\
& \frac{1}{16 \pi^{2}}\left\{\frac{1}{2} \varepsilon_{\alpha \beta \lambda \mu}\left[A_{\alpha \beta} A_{\lambda \mu}+\frac{1}{3} W_{\alpha \beta} W_{\lambda \mu}\right]\right. \\
& +\frac{16}{3} W_{\lambda} W_{\mu}\left(\partial_{\lambda} W_{\mu}\right)-\frac{4}{3} W^{2}(\partial \cdot W) \\
& \left.+\frac{2}{3} \partial^{2}(\partial \cdot W)\right\}
\end{aligned}
$$

The interesting observation is that the terms appearing in the first bracket are the same present in an $\eta=\pi / 4$ intermediate step in the continuous duality transformation for the lagrangian. This may suggest that a lagrangian density including both kinds of terms may improve as a dual symmetric version.

A last remark: Belyaev and Schapiro [42] have studied the axial current coupling as a remnant of torsion when general relativity is taken to the limit of flat space. It may be interesting to look whether this may be an indication of a natural Heaviside duality when gravity is taken into account

\section{A consistent duality scheme}

As I have shown, the strict concept of electromagnetic duality, implying the same field as produced from electric or magnetic charges, carries several problems and questions. Without monopoles, the theory has funny properties, ensuing mainly from the fact that Heaviside duality is not a symmetry of the lagrangian. The imposition of duality as a symmetry led Schwarz and Sen [16] to develop an ad hoc formalism apparently without relativistic invariance, and a generator for the transformation found by Deser and Teitelboim [14] is non-local in space. The latter results were obtained for the free field case. With two potentials, some of these features are tamed, but energy ceases to be positive under the transformation.

Recently, with C. A. P. Galvão we have looked to the problem [43]. The idea of consistency results in an heterodox proposal, abandoning the identity of electric and magnetic fields having different charges as sources. In the vacuum sector, the symmetry allows free exchange between electric and magnetic fields, in the Heaviside original fashion. However, symmetry under duality means here exchange between dissimilar fields. 
A dual theory to the usual electrodynamics, magnetodynamics would be:

$$
\left.\begin{array}{rl}
\nabla \cdot \mathbf{E}^{\prime} & =0 \\
\nabla \cdot \mathbf{B}^{\prime} & =\mu_{0} \rho_{m} \\
\nabla \wedge \mathbf{E}^{\prime} & =-\frac{1}{\epsilon_{0}} \mathbf{j}_{m}-\frac{\partial \mathbf{B}^{\prime}}{\partial t} \\
\nabla \wedge \mathbf{B}^{\prime} & =\frac{1}{c^{2}} \frac{\partial \mathbf{E}^{\prime}}{\partial t}
\end{array}\right\} .
$$

The primes indicate that the fields represented by the symbols do not satisfy the same equations as the ones appearing in the ordinary theory.

Potentials are introduced from the homogeneous equations:

$$
\left.\begin{array}{l}
\mathbf{E}^{\prime}=\nabla \wedge \mathbf{W}^{\prime} \\
\mathbf{B}^{\prime}=-\frac{\partial \psi^{\prime}}{\partial t}+\frac{1}{c^{2}} \frac{\partial \mathbf{W}^{\prime}}{\partial t}
\end{array}\right\} .
$$

In three-dimensional notation, the lagrangian density that produces the field equations above reads:

$$
\mathcal{L}\left\{\mathbf{B}^{\prime}, \mathbf{E}^{\prime}\right\}=\frac{\epsilon_{0}}{2}\left[c^{2} \mathbf{B}^{\prime 2}-\mathbf{E}^{\prime 2}\right]-\rho_{m} \psi^{\prime}-\mathbf{j}_{m} \cdot \mathbf{W}^{\prime}
$$

Written in covariant four-dimensional notation, one has to introduce a second rank antisymmetric tensor, $G_{\mu \nu}$ such that its time-space components are related to $c \mathbf{B}^{\prime}$ and the space-space ones to $\mathbf{E}^{\prime}$, and the corresponding four-vectors for the potentials and current density. This tensor is not to be taken as the dual of the electromagnetic tensor in the Maxwell case, the fields are assumed to be different.

Duality now corresponds to the exchanges

$$
\mathbf{E} \rightarrow-c \mathbf{B}^{\prime}, \quad c \mathbf{B} \rightarrow \mathbf{E}^{\prime}
$$

and the corresponding equations in the opposite direction.

A lagrangian density in four-dimensional notation covering electrodynamics and magnetodynamics with the above transformation properties is:

$$
\begin{aligned}
\mathcal{L} & =-\frac{\epsilon_{0}}{4} F^{\mu \nu} F_{\mu \nu}-\frac{\epsilon_{0}}{4} G^{\mu \nu} G_{\mu \nu} \\
& -\frac{1}{c} j^{\mu} A_{\mu}-\frac{1}{c} j^{\mu} W^{\prime}{ }_{\mu}+\text { (gauge fixing terms) } .
\end{aligned}
$$

This theory has the symmetry as required, possess a positive energy and the generator for the symmetry is

$$
M=\int d^{3} x\left[A^{\mu} \Pi^{\prime}{ }_{\mu}-W^{\prime \mu} \Pi_{\mu}\right]
$$

and is perfectly local and covariantly written. Here, $A^{\mu}$ and $\Pi_{\mu}$ are the usual potential and canonical momentum four-vectors of Maxwell theory, and $\Pi^{\prime}{ }_{\mu}=$ $-\epsilon_{0} c B^{\prime}{ }_{k} \delta_{k \mu}$.
Though the physical idea of relating different fields through a "duality" transformation may sound inappropriate, let us recall the important Hodge decomposition theorem for differential forms [44]: for a compact, oriented Riemannian manifold, any $p$-differential form may be decomposed in the sum

$$
\omega=\omega_{0}+d \alpha+\delta \beta
$$

where $\Delta \omega_{0}=0$, and each term in the sum belongs to a vector subspace in the space of $p$-differential forms orthogonal to the other two. This raises the question whether in the two potential theory it is legitimate to identify the two contributions to field-strength tensor as being equivalent physical fields.

\section{Duality and monopoles in non-abelian gauge theories and phenomenology}

In the last decades of evolution of the theory of elementary particles as expressed by non-abelian gauge theories through the "standard model", monopoles have played different rôles. I just want to mention some recent applications of the monopoles in non-abelian gauge theories which called my attention.

Let me first make a digression, and mention that $\mathrm{J}$. Simões showed to me that in 1979 Senjanovic was looking for a model with $S U(2)_{L} \otimes S U(2)_{R} \otimes U(1)$ symmetry for the electroweak interactions, which was consistent with the phenomenology data at the time [45]. The idea was that the right symmetry was broken via the usual HKBEA mechanism and the charged vector meson from the right part acquired a large mass.

Going back to the matter, the group at Rutherford Laboratory led by Chang Hong-Mo is studying nonabelian gauge theories in a differential geometric context [46]. In 1995, he, Tsou Sheung Tsun and Jacqueline Faridani proposed an extension of what is Hodge duality in abelian theories for non-abelian ones. This was to take advantage of the notion of duality, in the form originally visualized by Dirac. In Dirac terms [4], "the idea is so fine that one would be surprised if Nature had made no use of it".

Usually, one starts from a free action,

$$
S^{0}=S^{0}{ }_{F}+S^{0}{ }_{M}
$$

where in the right hand side each contribution corresponds to field and matter free actions.

Interaction is introduced adding an "interaction term ", whose form is deduced from phenomenology and/or invariance considerations. Chan and collaborators, instead, propose that matter and fields are determined to obey as a constraint that the dual field intensity has a magnetic monopole as a source. Take 
the example of the abelian theory with a Dirac field, the constraint reads:

$$
\partial_{\mu} * F^{\mu \nu}(x)=4 \pi \tilde{e} \tilde{\tilde{\Psi}}(x) \gamma^{\nu} \tilde{\Psi}(x)
$$

and the action includes the constraint with a Lagrange parameter field,

$$
S^{\prime}=S^{0}+\int d^{4} x \lambda_{\nu}\left\{\partial_{\mu} * F^{\mu \nu}-4 \pi \tilde{e} \tilde{\tilde{\Psi}}(x) \gamma^{\nu} \tilde{\Psi}(x)\right\}
$$

Notice that I would have used the axial current in the constraint.

Minimizing with respect to $F^{\mu \nu}(x)$ one gets

$$
F^{\mu \nu}=2 \pi \varepsilon^{\mu \nu \rho \sigma}\left(\partial_{\rho} \lambda_{\sigma}-\partial_{\sigma} \lambda_{\rho}\right),
$$

and with respect to $\overline{\tilde{\Psi}}(x)$,

$$
(i \not \partial-m) \tilde{\Psi}(x)=-4 \pi \tilde{e} \lambda_{\mu}(x) \gamma^{\mu} \tilde{\Psi}(x) .
$$

In fact, one gets,

$$
* F_{\mu \nu}=-4 \pi\left(\partial_{\mu} \lambda_{\nu}(x)-\partial_{\nu} \lambda_{\nu}(x)\right) \text {. }
$$

A new gauge invariance with the opposite parity is gained from $\lambda_{\mu}(x)$.

The coupling $\tilde{e}$ is related to the original one via the Dirac relation (in these units)

$$
e \tilde{e}=n / 4 \text {. }
$$

Personally, I can't see how they arrive to this conclusion for the spinor theory.

For the non-abelian theory, Chan, Tsou and Faridani obtained an extension of Hodge duality satisfying

- It reduces to Hodge duality in the abelian case

- Applied twice goes back to the original object (with possibly a phase factor)

- Non-abelian charges for the original fields are monopoles of the dual field

Duality is introduced via loop variables; the main feature is that, like the abelian case, one gets as a bonus an additional gauge invariance with the opposite parity.

The phenomenological applications provene from the breaking of this additional symmetry. Higgs fields are taken as the frame vectors of $S U(3)$, they belong to the fundamental representation; in the presence of monopoles they have to be adjusted for each chart.

The dual colour distinguish generations. In fact, the tree approximation for masses produces a mass for one generation, the others being massless (think in the mass relations $\tau: \mu$ : electron). They are then able to calculate the Cabibbo-Kobayashi-Maskawa matrix, and the result is rather impressive:

$$
\begin{array}{cl}
\left|V_{r s}\right|= & \\
\left(\begin{array}{ccl}
0,9755(0,9745-0,9757) & 0,2199(0,219-0,224) & 0,0044(0,002-0,005) \\
0,2195(0,218-0,014) & 0,9746(0,9736-0,9750) & 0,0452(0,036-0,046) \\
0.0143(0,004-0,014) & 0,0431(0,034-0,046) & 0,9990(0,9989-0,9993)
\end{array}\right)
\end{array}
$$

The figures in parenthesis are the phenomenological values. The values for the masses are not so good, but Chan believes that, since the calculations involve extrapolations in a logarithmic scale, they should be less reliable.

Another development is due to Tanmay Vachaspati and collaborators [47]. The idea is that may be the fermions of the standard model may be seen as the monopoles of a dual bosonic Grand Unification Theory. Starting from a $S \tilde{U}(5)$ model, successive breakings leads to the dual of usual electromagnetism, $U \tilde{U(1)}$. The breaking scheme is

$$
\begin{aligned}
S \tilde{U}(5) & \rightarrow\left[S \tilde{U}(3) \otimes S \tilde{U}(2) \otimes U \tilde{U}(1)^{\prime}\right] / Z_{6} \\
& \rightarrow[S \tilde{U}(3) \otimes U \tilde{(1)}] / Z_{3} \\
& \rightarrow U \tilde{(1)} .
\end{aligned}
$$

A monopole charge is obtained from the homotopy groups for each group in the chain:

$$
Q^{(n)}=n_{3} Q_{3}+n_{2} Q_{2}+n_{1} Q_{1}
$$

and stability requires $n= \pm 1, \pm 2, \pm 3, \pm 4, \pm 6$, with $n_{1}=n(\bmod .3), n_{2}=n(\bmod .2)$ and $n_{3}$ an integer.

The assignments for the known particles result:

$$
\left.\begin{array}{rlc}
(u, d)_{L} & \rightarrow & n=+1 \\
d_{R} & \rightarrow & n=-2 \\
(\nu, e)_{L} & \rightarrow & n=-3 \\
u_{R} & \rightarrow & n=+4 \\
e_{R} & \rightarrow & n=-6 .
\end{array}\right\}
$$

It has been shown that diquarks are unstable, whereas clusters of three quarks are stable. The prob- 
lem of spin has been attacked too, taking profit from the "spin from isospin" construction.

The more recent work on this approach by Lepora [47] converges to the views by Olive and collaborators and Chan and collaborators.

Even in case the final goal is not attained, one may ask whether these figures quoted from the works by Chan, Tsou and collaborators and Vachaspati and followers are mere funny numerical accidents or point to the true structure of the theory taken for the "standard model" . In any case, in my opinion they open interesting pathways for research.

\section{Conclusion}

In this swift glance on the subject, I hope to have made clear that at least two different views of it are referred in the same terms. One, pure electromagnetic Heaviside duality, does not include necessarily magnetic monopoles. The answer to the first question addressed at the end of section 2 is then on the negative.

The second view introduces the notion of duality from two-dimensional spacetime Sine-Gordon and Thirring model relationship. There seems to be fruitful results of this ideas in nonabelian theories if one takes for the solitons in this approach the magnetic monopoles.

In my view, the pure Heaviside duality may be implemented either by imposing the identity of the fields generated by electric or magnetic charges, or extending the phase space to include different fields for each different kind of charge. In the first case, the problems that appear are related to the fact that the transformation is not a symmetry of the action, but of its Euler-Lagrange equations. One may overcome these difficulties through a time inversion in the quantized version, but it remains to see if the final theory is consistent, and so remains an open question which is the right path to follow.

I hope to have set clear that Heaviside duality is a physical condition on fields, potentials and currents. Hodge duality, while being a useful tool to write the results in a concise form, is just a geometrical setting.

In any case, it remains to show that duality may produce meaningful physical results in a field theoretical framework. Coming back to the example of the hydrogen atom, is not clear that we have an spectroscope with enough resolution to put into evidence a symmetry under the broken theory leading to the Maxwell equations. The results obtained by the groups implementing the idea in nonabelian theories open a window for hope.

\section{Acknowledgements}

I wish to thank Clóvis Wotzasek and Franciscus Vanhecke for his kind reading of the original text, and their criticism and suggestions.

\section{References}

[1] J. H. Schwarz and N. Seiberg, Rev. Mod. Phys. 71(1999) special issue, S112.

[2] G. Heaviside, Phil. Trans. Roy. Soc.(London) A 183, 423 (1893).

[3] I. Larmor, Collected Papers, London(1928); G. I. Rainich, Trans. Am. Math. Soc. 27, 106 (1925).

[4] P. A. M. Dirac, Proc. Roy. Soc. A133, 60 (1931).

[5] P. J. Mohr and B. N. Taylor, Rev. Mod. Phys. 72, 351 (2000).

[6] W. Greub and H.-R. Petry, J. Math. Phys. 16, 1347 (1975).

[7] C N. Yang and T. T. Wu, Nucl. Phys. B107, 365 (1976); Phys. Rev. D14, 437 (1976).

[8] P. A. M. Dirac, Phys. Rev. 74, 817 (1948).

[9] A. Nisbet, Proc. Roy. Soc. A231, 250 (1955).

[10] N. Cabibbo and E. Ferrari, Il Nuovo Cim. 23, 1147 (1962).

[11] F. Röhrlich, Phys. Rev. 150, 1104 (1966).

[12] J. Schwinger, Phys. Rev. 144, 1087 (1966); ibídem151, 1048 (1966), 151, 1055 (1966), 173, 1536 (1968).

[13] D. Zwanziger, Phys. Rev. D 3, 380 (1971).

[14] S. Deser and C. Teitelboim, Phys. Rev. D13, 1592 (1976).

[15] C. Montonen and D. Olive, Phys. Lett. B72, 117 (1977).

[16] J. Schwarz and A. Sen, Nucl. Phys. B411, 35 (1994).

[17] N. Seiberg and E. Witten, Nucl. Phys. B431, 484 (1994).

[18] B. Mc Clain, Y. S. Wu and F. Yu, Nucl. Phys. B343, 689 (1990); C. J. Wotzasek, Phys. Rev. Lett. 66, 129 (1991);I. Martin and A. Restuccia, Phys. Lett. B323, 311 (1994); F. P. Devecchi and M. Henneaux, Phys. Rev. D54, 1606 (1996); N. Berkovitz, Phys. Lett. B388, 743 (1996)

[19] P. Pasti, D. Sorokin and M. Tonin, Phys. Lett. B 352 , 59 (1994); Phys. Rev. D56, 2473 (1997).

[20] R. L. Pakman, Phys. Lett. B 474, 309 (2000), and hepth/9912057.

[21] H. O. Girotti, Phys. Rev. D55, 5136 (1997).

[22] H. Girotti, M. Gomes, V. Rivelles and A. J. da Silva, Phys. Rev. D56, 6615 (1997).

[23] C. Wotzasek, Phys. Rev. D59, 125026 (1998).

[24] D. Singleton, Am. J. of Phys.64, 452 (1996).

[25] W. A. Barker and F. Graziani, Am. J. Phys 46, 1111 (1948); Phys. Rev. D18, 3849 (1978).

[26] C. A. P. Galvão and J. A. Mignaco, Proceedings X "J. A. Swieca" Summer School of Particles and Fields, Águas de Lindóia, (1999).

[27] M. Y. Han and L. C. Biedenharn, Il Nuovo Cim. A2, 544 (1971).

[28] J. D. Jackson, Classical Electrodynamics, 3rd. Ed., J. Wiley and Sons, Inc. (1998), p. 273. 
[29] D. I. Olive, Exact electromagnetic duality,in "Cargèse 1997, Strings, branes and dualities", 3; Lectures in electromagnetic duality II, in "Campos de Jordão 1997, Particles and Fields", 166.

[30] M. A. C. Kneipp, Introduction to dualities in gauge theories, X "J. A. Swieca Summer School for Particles and Fields", Águas de Lindóia (1999).

[31] P. Bocchieri and A. Loinger, Il Nuovo. Cim. A47, 475 (1978).

[32] P. C. R. Cardoso de Mello, S. Carneiro and M. C. Nemes, Phys. Lett. B384, 197 (1996).

[33] R. Dijkgraaf, lectures delivered at the Les Houches School on Theoretical Physics, Session LXIV: Quantum Symmetries, Les Houches, France, (1995).

[34] S. Coleman, Phys Rev. D11, 2088 (1975).

[35] S. Mandelstam, Phys. Rev. D11, 3026 (1975).

[36] P. W. Anderson, Phys. Rev. 130, 439 (1963); P.W. Higgs, Phys. Rev. 145, 1156 (1966); T. W. Kibble, Phys. Rev. 155, 1554 (1967); R. Brout and F. Englert, Phys. Rev Lett. 13, 321 (1964)

[37] L. H. Ryder, J. Phys. A 13, 437 (1980).

[38] J. Schwinger, Phys. Rev. 144, 1087 (1965).

[39] C. Jayewardena, Helv. Phys. Acta 61, 636 (1988).

[40] J. A. Mignaco, C. Sigaud, A. R. da Silva and F. Vanhecke, Rev. Math. Phys. 9, 689 (1997).
[41] A. P. Balachandran, G. Marmo, V. P. Nair and C. G. Trahern, Phys. Rev. D 25, 2713 (1982).

[42] A. S. Belyaev and I. L. Schapiro, Phys. Lett. B 425, 246 (1998); Nucl. Phys. B543, 20 (1999).

[43] C. A. P. Galvão and J. A. Mignaco, hep-th/0002182 (2000).

[44] S. Rosenberg, The Laplacian on a Riemannian Manifold, Cambridge U. Press (1997).

[45] G. Senjanovic, Nucl. Phys. B153, 334 (1979), and references therein

[46] Chan Hong-Mo and Tsou Sheung Tsun, Some Elementary Gauge Theory Concepts, World Sci. Publ. Co. Ltd., Singapore (1995); C. H.-M., Yang-Mills duality and the generation puzzle, hep-th/0007016, where most of the bibliography is found; C.H.-M., T.S.T. and Jacqueline Faridani, Phys. Rev. D52, 6143 (1995), ibídem D51, 7040 (1995); C.H.-M. and T.S.T., Phys. Rev. D56, 3646 (1997), ibídem D57, 2507 (1998).

[47] T. Vachaspati, Phys. Rev. Lett. 76, 188 (1996); T. V. and Hong Liu, Phys. Rev. D56, 1300 (1997); T. V. with Hong Liu and G. D. Starkman, Phys. Rev. Lett. 78, 1223 (1997), and Phys. Lett. B427, 1223 (1998); N. F. Lepora, hep-ph/9910493, hep-ph/0008323. 